\section{AB1419-HPR EFFICIENCY OF INDIVIDUAL STEEL ORTHESIS IN} HEAVEN SPRAY

Dmitry Baryshov ${ }^{1}$, Evgenia Guryanova ${ }^{2} .{ }^{1}$ Chuvash State University, Cheboksary, Russian Federation; ${ }^{2}$ Chuvash State University, Rheabilitation, Cheboksary, Russian Federation

Background: The most common cause of heel pain is the heel spur, which is $75 \%$ of patients with heel pain. The intensity of the pain does not depend on the size of the spur, as determined on the radiograph. Often sharp in shape and large in size, the spurs are a random X-ray finding. At the same time, severe pains in the heel region are possible with a normal radiograph [1]..

Objectives: The purpose of this study is to establish the effectiveness of the treatment of patients with pain syndrome in the heel spur, by affecting the main components of the pathogenesis of individually manufactured orthopedic insoles.

Methods: During the year, under our supervision there were 156 patients aged from 32 to 67 years. Gender composition - 123 (79\%) - women, and $32(21 \%)$ - men. The average time between the onset of clinical symptoms and the first day of treatment was 134 days. The average spur size was $4.3 \mathrm{~mm}$. In $80 \%$ of patients, a history of pain lasted for more than 3 months. Patients were divided into 2 groups. The experimental group (G1) used individually manufactured insoles (78 people: 61 - women, 17 - men). The control group (G2) used factory insoles (78 people: 58 - women, 20 - men). 152 patients (98\%) completed the protocol: $78(100 \%)$ in $\mathrm{G} 1$ and $74(96 \%)$ in G2. The criterion for inclusion in the study was the diagnosis of the heel spur. The diagnosis was made based on anamnesis, ultrasound of the plantar aponeurosis, and radiography of the heel region. The exclusion criteria were patients with heel spurs without heel pain, as well as systemic diseases: rheumatoid arthritis, diabetes mellitus, and severe vascular pathology.

The degree of longitudinal flat-footedness, stability of the foot, individual deformities of the feet were clinically evaluated using clinical and functional methods and the method of vacuum trace modeling [2].

Individual insoles were made based on frame materials in the projection of the arches of the foot. Dense materials maintain a constant tension of the plantar aponeurosis. G1 patients were made full contact insoles with a correction of the transverse arch, without adding relief elements under the heel.

The control group used factory-made insoles from different manufacturers, with soft elements under the heel. Measurement parameters: average pain intensity on a VAS scale; duration of pain throughout the day; distance traveled; subjective comfort when using insoles. The observation period is 12 calendar months.

Results: The average VAS score in G1 decreased from 5.40 .3 at the beginning of the study to 1.01 in 62 patients $(81 \%) .15$ patients reported pain reduction, the average VAS score was 2.10 .3 .

Complete relief of $\mathrm{G} 1$ pain has been reported in patients with an osteophyte size of less than $5 \mathrm{~mm}$. G1 patients regained their usual movement distance of $3.9 \mathrm{~km}$ per day. Cases of recurrence of heel pain is not recorded. No patients experienced discomfort from the use of insoles throughout the entire period of adaptation.

In G2, the average VAS score decreased from 5.60 .3 at the beginning of the study to 2.90 .3 . Patients of the G2 group were able to increase the distance of movement to $1.2 \mathrm{~km}$ per day.

Conclusion: When using individual insoles, $93 \%$ of patients reported complete relief of pain. $7 \%$ of patients noted a significant reduction in pain. The use of individual insoles in the treatment of plantar fasciitis made it possible to reduce pain in a shorter time.

\section{REFERENCES}

[1] Kotelnikov G.G. Traumatology and Orthopedics. M.: GEOTAR-Med, 2018. $776 \mathrm{p}$.

[2] Joint pain/K. V. Kotenko (and others). M.: GEOTAR - Med, - 2018. 560 p.

Disclosure of Interests: None declared

DOI: 10.1136/annrheumdis-2019-eular.8007

\section{AB1420-HPR THE USE OF SUBCUTANEOUS METHOTREXATE IN INFLAMMATORY ARTHRITIS: TRANSLATING RESEARCH INTO PRACTICE USING QUALITY IMPROVEMENT METHODOLOGY.}

\section{Aicha Bouraoui1'. 'Basildon University Hospital, Basildon, United Kingdom}

Background: Methotrexate (Mtx) is considered as the drug of choice in the management of inflammatory arthritis (IA). There is growing evidence of the benefit of sub-cutanous $(\mathrm{s} / \mathrm{c})$ methotrexate compared to the oral route. At the same dose, pharmacokinetic studies showed that s/c Mtx has better bioavailability. RCT studies showed that patients respond better to s/c mtx. Finally s./c Mtx has better tolerability with less risks of $\mathrm{Gl}$ side effects.

Translating research into practice can be sometimes challenging due to a number of barriers including individual choices and variations as well as systems or organizational factors. Over the recent years there has been great focus on quality improvement in healthcare to make health care "safe, effective, patient centered timely efficient and equitable".

Objectives: We aimed that within 6 months period, $100 \%$ of our patients with inflammatory arthritis should have trial of $\mathrm{s} / \mathrm{c}$ methotrexate before starting biologics unless there is contraindication.

Methods: We reviewed the data of 50 patients to assess management of patients with IA using our biologic register. We used the 5 why strategy to have better understanding of the lack of prescribing of $\mathrm{s} / \mathrm{c} \mathrm{mtx}$ and vairiations in clinical practice. By applying improvement science we standardized our pathway, included methotrxate s/c trial prior to biologics. We also conducted a number of interventions including multidisciplinary educational events and one to one meetings, we mobilised and organised our resources in better way to meet our patients needs. We monitored our data over time and reviewed our practice accordingly.

Results: Baseline data showed that the majority of our patients had rheumatoid arthritis (RA) (74\%) followed by Psoriatic arthritis in $24 \%$ of cases. Most patients had initial trial of methotrexate but only $32 \%$ were kept on oral methotrexate and $26 \%$ of patients had trial of $\mathrm{s} / \mathrm{c} \mathrm{mtx}$ before starting biologics. The main indication of starting $\mathrm{s} / \mathrm{c}$ Mtx was oral methotrexate induced gastrointestinal side effects. Methotrexate was stopped all together in $34 \%$ of patients due to suspected major side effects(SE). SE mainly encountered with oral route with $10 \%$ suspected ILD, $14 \%$ had deranged LFTs. In order to have better understanding with issues related to the poor use of subcut Mtx the author conducted staff unstructured interviews to explore their experience with $\mathrm{s} / \mathrm{c} \mathrm{Mtx}$. We also noticed variations between clinicians in their clinical practices.

Our theory of change included a number of interventions and results showed significant improvement in prescribing of $\mathrm{s} / \mathrm{c}$ Mtx among IA patients. 3 months after starting the projects 1 st cycle: 42 patients were switched to sub-cut Mtx with only 6 patients required subsequent biologics use.

There was reduction in the number of patients referred for biologics and among those started on biologics $50 \%$ of patients had trial of S/C methotrexate.

Conclusion: In summary, our baseline data showed poor use of s/c Mtx in patients with IA despite the growing evidence of its benefit. In order to translate research findings into practice we used 5 whys methodology to have better understanding of the barriers within our systems, we applied QI methodology and standardized our practice for better use of resources at lower cost.

\section{REFERENCES}

[1] Braun J, Kastner P, Flaxenberg P, et al. Comparison of the clinical efficacy and safety of subcutaneous versus oral administration of methotrexate in patients with active rheumatoid arthritis: results of a six-month, multicenter, randomized, double-blind, controlled, phase IV trial. Arthritis Rheum 2008;58:7381.

[2] Stamp LK, Barclay ML, ODonnell JL, et al. Effects of changing from oral to subcutaneous methotrexate on red blood cell methotrexate polyglutamate concentrations and disease activity in patients with rheumatoid arthritis. $J$ Rheumatol 2011;38:25407.

Acknowledgement: Dr Bharadwaj and the Rheumatology team at Basildon Hospital.

Disclosure of Interests: None declared

DOI: 10.1136/annrheumdis-2019-eular.8298

\section{AB1421-HPR VIRTUAL CLINICS IN THE PRESENT- A PREDICTOR FOR THE FUTURE?}

Tina Parish', Maathu Ratnaraj', Tazeen J. Ahmed ${ }^{2} .{ }^{1}$ St George's University of London, London, United Kingdom; ${ }^{2}$ Croydon University Hospital, London, United Kingdom

Background: There is great interest in non face to face (F2F), internet or app based outpatient interact at the moment. As these become established, we look at the non F2F appointments already happening in telephone and virtual clinics in a busy urban rheumatology department. Here we look at the type of diagnoses dealt with in a non F2F environment and potential outcomes from these non F2F appointments. 